C-A/AP/\#172 September 2004

\title{
Design of an Interaction Region for the Electron-Light Ion Collider ELIC
}

\author{
Christoph Montag
}

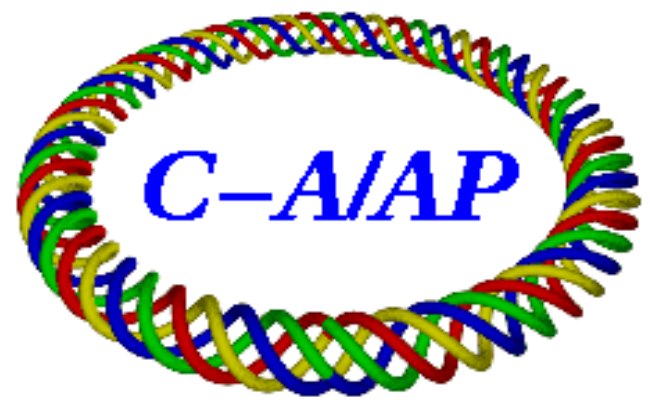

Collider-Accelerator Department Brookhaven National Laboratory Upton, NY 11973 


\title{
Design of an Interaction Region for the Electron-Light Ion Collider ELIC
}

\author{
Christoph Montag \\ Brookhaven National Laboratory, Upton, NY 11973
}

\begin{abstract}
The Electron-Light Ion Collider ELIC proposed by Jefferson Lab aims at a luminosity in the $10^{35} \mathrm{~cm}^{-2} \mathrm{sec}^{-1}$ range for collisions of $150 \mathrm{GeV}$ protons on $7 \mathrm{GeV}$ electrons $[1,2]$. To achieve these high luminosities, very strong low- $\beta$ focusing of low-emittance beams is required. The interaction region design concept described in Ref. [2] consists of superconducting quadrupole triplets that focus the hadron beam to $\beta$-functions of $\beta^{*}=$ $5 \mathrm{~mm}$ in both planes at the interaction point (IP). This scheme requires quadrupoles with peak fields of $9 \mathrm{~T}$ to ensure a minimum aperture of $6 \sigma_{h}$, where $\sigma_{h}$ is the rms beam size of the hadron beam.

In this paper we present an alternative interaction region design based on superconducting quadrupole doublets that provide unequal $\beta$-functions in the two planes at the IP. The advantage of this scheme is that it requires peak quadrupole fields of only $6.3 \mathrm{~T}$ for a minimum aperture of $12 \sigma_{h}$, while potentially doubling the luminosity.
\end{abstract}

\section{Introduction}

To provide the worldwide nuclear physics community with a high-luminosity electron-ion collider with a center-of-mass energy in the 20 to $65 \mathrm{GeV}$ range, Jefferson Lab has proposed the CEBAF-based Electron-Light Ion Collider ELIC. This machine consists of a figure- 8 shaped polarized ion storage ring with maximum proton energy of $150 \mathrm{GeV}$, and a similarly shaped $7 \mathrm{GeV}$ electron circulator ring. Both beams are brought into collision in four interaction regions located in the straight sections. To achieve high luminosities in the $10^{35} \mathrm{~cm}^{-2} \mathrm{sec}^{-1}$ range, low- $\beta$ focussing of low-emittance beams to $\beta$-functions of $\beta^{*}=5 \mathrm{~mm}$ in both planes at the interaction point (IP) is required, together with a bunch collision rate of $1.5 \mathrm{GHz}$. Table 1 lists some basic parameters of ELIC according to Ref. [2].

Since a bunch spacing of only $20 \mathrm{~cm}$ is foreseen, a large crossing angle of $100 \mathrm{mrad}$ is mandatory to avoid parasitic collisions [1]. This simplifies the interaction region design, because no dipole fields are required to separate the beams that would generate intense synchrotron radiation in the close vicinity of the interaction point (IP). Additionally, the use of common magnets for both 


\begin{tabular}{lcc}
\hline \hline parameter & EPAC $[2]$ & "NEW" \\
\hline energy E $(\mathrm{p} / \mathrm{e})[\mathrm{GeV}]$ & $150 / 7$ & $150 / 7$ \\
\# particles/bunch $\mathrm{N}(\mathrm{p} / \mathrm{e})\left[10^{10}\right]$ & $0.4 / 1.0$ & $0.4 / 1.0$ \\
collision rate $[\mathrm{GHz}]$ & 1.5 & 1.5 \\
emittance $\epsilon(\mathrm{x} / \mathrm{y})[\mathrm{nm}]$ & $6.3 / 0.25$ & $6.3 / 0.25$ \\
$\beta^{*}(\mathrm{x} / \mathrm{y})[\mathrm{mm}]$ & $5 / 5$ & $25 / 5$ \\
$\sigma^{*}(\mathrm{x} / \mathrm{y})[\mu \mathrm{m}]$ & $5.8 / 1.2$ & $13.0 / 1.2$ \\
beam-beam tuneshift $\xi_{h}(\mathrm{x} / \mathrm{y})$ & $0.002 / 0.01$ & $0.002 / 0.005$ \\
beam-beam tuneshift $\xi_{e}(\mathrm{x} / \mathrm{y})$ & $0.017 / 0.086$ & $0.019 / 0.043$ \\
\hline luminosity $\mathcal{L}\left[10^{34} \mathrm{~cm}^{-2} \mathrm{sec}^{-1}\right]$ & 7.7 & 3.4 \\
\hline
\end{tabular}

Table 1: Parameters of the Electron-Light Ion Collider ELIC. The column labeled "EPAC" shows the design parameters presented in Ref. [2], while the parameters used for the design presented in this paper are listed in the column labeled "NEW". Luminosities are given for equal bunch intensities in both designs. However, in the "NEW" design, bunch currents can potentially be doubled due to the smaller beam-beam tuneshift resulting from unequal $\beta$-functions in the two planes.

beams is unnecassary because the separation between the two beams is large enough to guide either one of the beams through a field-free region of the focusing elements for the other beam, even at the innermost quadrupole which is $2 \mathrm{~m}$ from the IP.

\section{Design considerations and optics}

A novel design feature of ELIC is the use of unequal hadron beam emittances in the two transverse planes (see Table 1). This results naturally from intra-beam scattering and electron cooling in a flat, (locally) decoupled storage ring.

Focussing these beams to the same $\beta$-functions in both planes at the IP, $\beta_{x}^{*}=\beta_{y}^{*}$, results in very different beam-beam tuneshift parameters in the two planes, $\xi_{y} \gg \xi_{x}$. Since in the absence of single-bunch instabilities the maximum bunch intensity is limited by the larger beam-beam tuneshift parameter $\xi_{y}$ regardless of the value of $\xi_{x}$, a more balanced situation of the two planes is desirable. With the beam-beam tuneshift parameter given as

$$
\xi_{x, y}=\frac{N r_{c} \beta_{x, y}^{*}}{2 \pi \gamma \sigma_{x, y}\left(\sigma_{x}+\sigma_{y}\right)},
$$

where $N, r_{c}$, and $\gamma$ denote the number of particles in the oncoming beam, the classical particle radius and the Lorentz factor, respectively, this can be accomplished by increasing the horizontal $\beta$-function $\beta_{x}^{*}$. To keep the sizes of electron and hadron beam at the IP identical, this has to be applied to both beams. Since this also increases the horizontal beam size $\sigma_{x}$ of the oncoming 


\begin{tabular}{lcc}
\hline \hline parameter & QH1 & QH2 \\
\hline length $l[\mathrm{~m}]$ & 1.2 & 3.0 \\
strength $k\left[\mathrm{~m}^{-2}\right]$ & -0.5 & 0.12 \\
distance from IP $s[\mathrm{~m}]$ & 2.0 & 5.0 \\
$\hat{\beta}_{x}[\mathrm{~m}]$ & 677 & 5700 \\
peak field $\hat{B}[\mathrm{~T}]$ & 6.2 & 4.3 \\
\hline
\end{tabular}

Table 2: Hadron low- $\beta$ quadrupole doublet parameters.

beam, this reduces the vertical beam-beam tuneshift parameter $\xi_{y}$ while the horizontal tuneshift remains practically constant for $\sigma_{x} \gg \sigma_{y}$.

In the design presented here, $\beta$-functions are set to $\beta_{x}^{*}=25 \mathrm{~mm}$ horizontally and $\beta_{y}^{*}=5 \mathrm{~mm}$ vertically. In contrast to the triplet focussing scheme described in Ref. [2], these unequal $\beta$-functions require focussing by a low- $\beta$ quadrupole doublet, shown in Figure 1.

Since the vertical $\beta$-function $\beta_{y}^{*}$ is five times smaller than the horizontal one, the first lens of the doublet, located $2 \mathrm{~m}$ from the IP, focusses vertically. Its length needs to be kept short to avoid aperture problems due to the rapidly growing, defocused horizontal $\beta$-function. With a length of $l=1.2 \mathrm{~m}$, a strength of $k=-0.5 \mathrm{~m}^{-2}$ and a maximum horizontal $\beta$-function of $\hat{\beta}_{x}=677 \mathrm{~m}$ inside the magnet, the peak field is $6.2 \mathrm{~T}$, providing a minimum aperture of $12 \sigma_{h}$.

The second lens focuses horizontally, with a maximum horizontal $\beta$-function inside the lens of $\hat{\beta}_{x}=5750 \mathrm{~m}$. With a length of $l=3.0 \mathrm{~m}$ and a strength of $k=0.12 \mathrm{~m}^{-2}$, this magnet has a peak field of $4.3 \mathrm{~T}$ for $12 \sigma_{h}$ minimum aperture. Table 2 lists the parameters of the hadron doublet.

The spacing between the two hadron quadrupoles provides sufficient space for the electron low- $\beta$ doublet, which is depicted in Figure 1. The first, vertically focussing magnet of this doublet is $l=0.6 \mathrm{~m}$ long and has a strength of $k=$ $1.4 \mathrm{~m}^{-2}$. With the maximum horizontal $\beta$ function inside that magnet being $\hat{\beta}_{x}=929 \mathrm{~m}$, the peak field of this magnet is $1.6 \mathrm{~T}$ for a minimum aperture of $20 \sigma_{e}$.

The second, horizontally focussing magnet of the electron doublet is $l=0.7 \mathrm{~m}$ long and has a strength of $k=1.1 \mathrm{~m}^{-2}$. Together with a maximum horizontal $\beta$-function of $\hat{\beta}_{x}=2150 \mathrm{~m}$, a minimum aperture of $20 \sigma_{e}$ can be accomplished by a peak field of $1.9 \mathrm{~T}$. The parameters of the electron low- $\beta$ doublet are listed in Table 3 .

\section{Synchrotron Radiation}

Due to the absence of dipoles in the detector vicinity, synchrotron radiation in this area is only generated in quadrupoles. The core of the beam (where the majority of the electrons is located) experiences only relatively low magnetic fields 

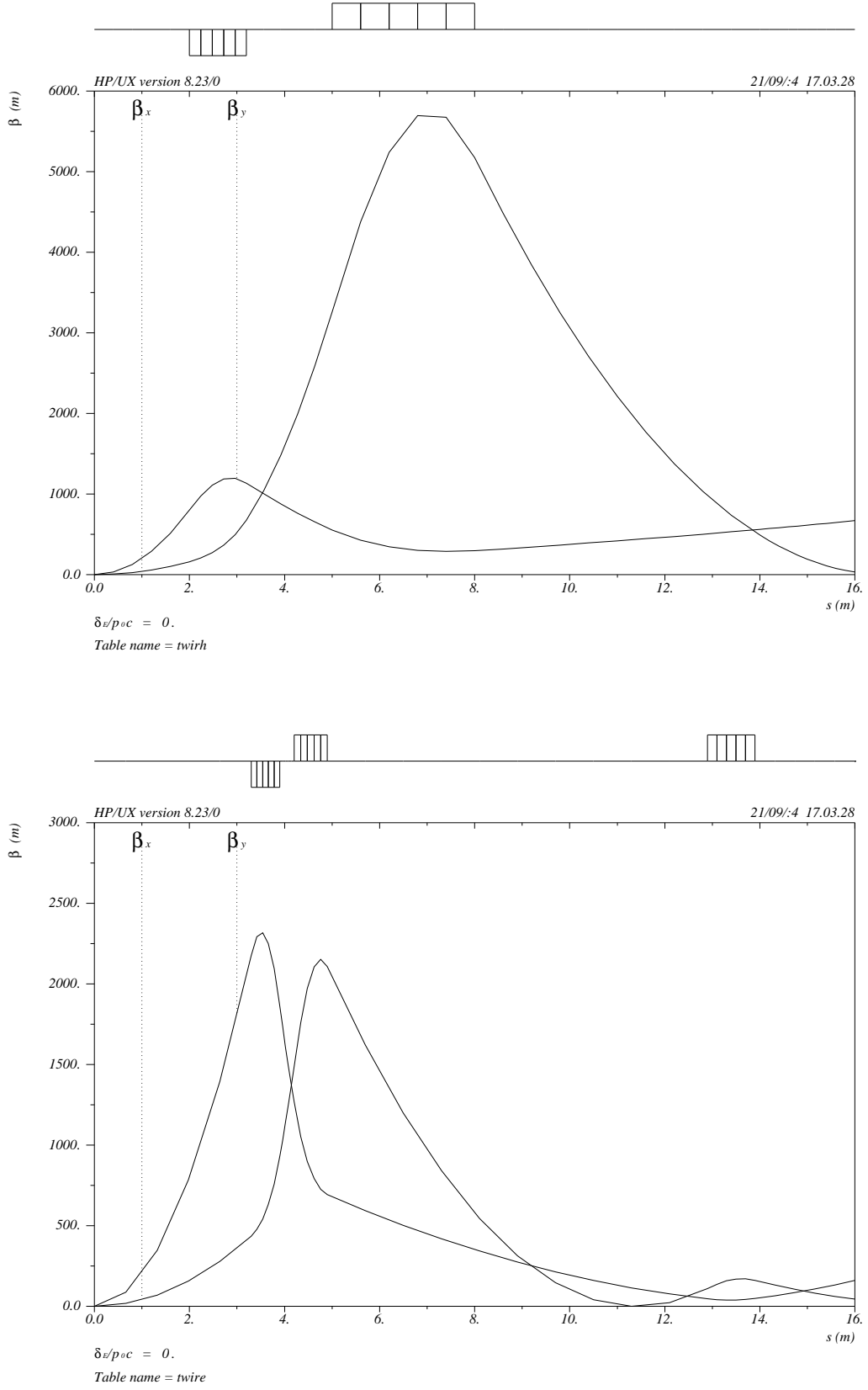

Figure 1: Hadron (top) and electron (bottom) interaction region lattices. The third magnet shown in the electron lattice indicates how this interaction region can be matched to the regular optics of the arc. 


\begin{tabular}{lcc}
\hline \hline parameter & QE1 & QE2 \\
\hline length $l[\mathrm{~m}]$ & 0.6 & 0.7 \\
strength $k\left[\mathrm{~m}^{-2}\right]$ & -1.4 & 1.1 \\
distance from IP $s[\mathrm{~m}]$ & 3.3 & 4.2 \\
$\hat{\beta}_{x}[\mathrm{~m}]$ & 929 & 2153 \\
peak field $\hat{B}[\mathrm{~T}]$ & 1.6 & 1.9 \\
\hline
\end{tabular}

Table 3: Parameters of the electron low- $\beta$ quadrupole doublet.

and therefore generates only soft photons. For example, electrons having a $1 \sigma_{e}$ amplitude experience magnetic fields of $0.1 \mathrm{~T}$, which produces synchrotron radiation photons with a critical energy of $E_{c}=3.3 \mathrm{keV}$. A small number of electrons at an amplitude of $20 \sigma_{e}$ in the transverse tails, however, undergoes focussing by magnetic fields up to some $2 \mathrm{~T}$, thus generating $E_{c}=65 \mathrm{keV}$ photons.

Since the total synchrotron radiation power generated by electrons in the transverse tails is quite small due to the small fraction of electrons at large amplitudes, this portion of the synchrotron radiation fan can be easily collimated upstream of the detector, thus protecting the detector from hits by high-energy photons. These collimators will be placed where the horizontal beam size is small while the fan is wide, thus ensuring sufficient free aperture for the beam itself.

Synchrotron radiation photons generated by the core of the beam stay mostly within the $20 \sigma_{e}$ electron beam itself and therefore do not hit any detector components. This is illustrated in Figure 2, which depicts the $20 \sigma_{e}$ electron beam envelopes and the corresponding synchrotron radiation fans generated by the $5 \sigma_{e}$ electron beam core, in both the horizontal and vertical planes.

In the vertical plane, the fan generated upstream of the IP can easily be collimated right at the entrance of the central detector, at $s=-2.0 \mathrm{~m}$, because at this location the $20 \sigma_{e}$ electron beam is narrower than the fan from the $5 \sigma_{e}$ beam. The innermost, vertically focusing low- $\beta$ quadrupole generates a fan that is somewhat outside the $20 \sigma_{e}$ beam on the outgoing side at distances beyond about $4 \mathrm{~m}$, but still well within the aperture of the corresponding magnet.

In the horizontal plane, however, the situation is somewhat more difficult. The strong focusing of the second, horizontally focusing quadrupole generates a synchrotron radiation fan with a "waist" around $s=-2 \mathrm{~m}$. This fan becomes very wide on the outgoing side and does not easily fit into the quadrupole magnet apertures there. Possible remedies are larger magnet apertures, which are feasible due to the relatively low gradient of these magnets, and/or installation of a synchrotron radiation collimator at $s=-2 \mathrm{~m}$ that also reduces the free aperture for the electron beam to some $10 \sigma_{e}$. This aperture reduction is not expected to have any negative effects because the electron beam is circulating for a few hundred turns at most. To avoid avoid additional detector background from electrons scattering off this collimator, an additional beam collimation system away from the IPs is advisable that defines the overall free beam aperture 
in the machine.

\section{Conclusion}

An alternative interaction region design for the Electron-Light Ion Collider ELIC has been presented. Using unequal $\beta$-functions in the two transverse planes at the interaction point, this scheme provides about half the luminosity of the design outlined in [2], given the same bunch intensities. The magnetic fields of the superconducting low- $\beta$ quadrupoles for the hadron beam are significantly lower than in the previous design [2], while simultaneously doubling the aperture in terms of rms beam size.

Additionally, this scheme leads to beam-beam parameters that are roughly a factor two below those of the original design [2]. Assuming that this is the only limitation, this allows to double the bunch intensities of both the electron and the hadron beam, thus approximately doubling the luminosity of the previous design [2] to $13.6 \cdot 10^{34} \mathrm{~cm}^{-2} \mathrm{sec}^{-1}$.

Doubling the bunch intensities also allows for doubling the $\beta$-functions at the IP, thus reducing the field strengths of the low- $\beta$ quadrupoles while providing the same luminosity as in the previous approach [2].

\section{Acknowledgments}

I would like to thank Lia Merminga, Slava Derbenev, Alex Bogacz and Rolf Ent for the opportunity to work on this design at Jefferson Lab, as well as for their hospitality and stimulating discussions during my visit. I am indebted to Todd Satogata for reading the manuscript.

\section{References}

[1] Ya. Derbenev, ICFA Beam Dynamics Newsletter, No. 30, 2003

[2] Ya. Derbenev et al., "Electron-Ion Collider at CEBAF: New Insights and Conceptual Progress", Proc. EPAC 2004 

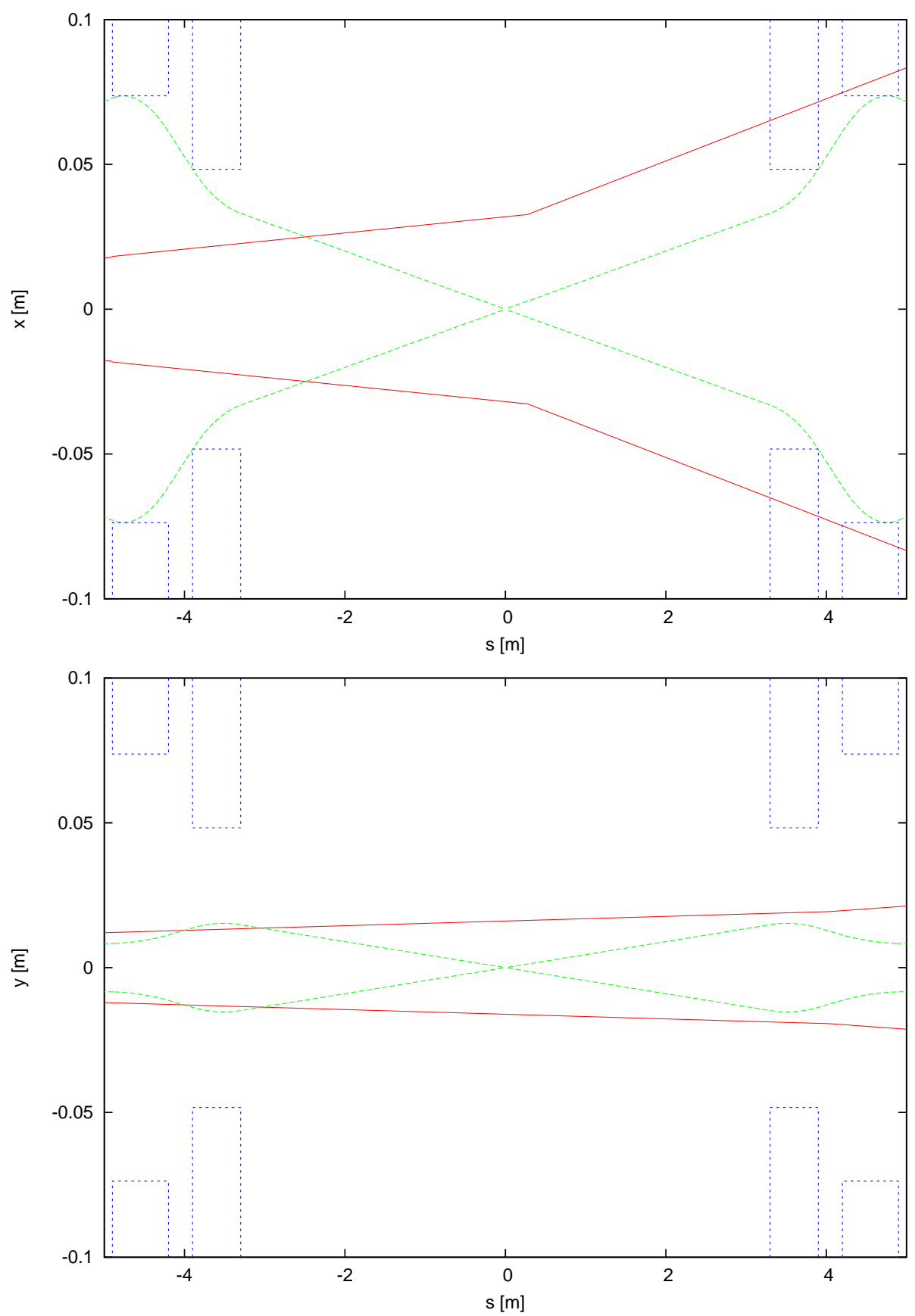

Figure 2: $20 \sigma$ beam envelopes (green) and synchrotron radiation fans as generated by particles at $5 \sigma$ (red). The superconducting low- $\beta$ magnets are indicated in blue. 